\title{
A Method of Optimization of Linear Observations for the Kalman Filter based on a Generalized Water Filling Theorem
}

\author{
Yoshiki TAKEUCHI and Akihiro HIRATA \\ Dept. of Information Science, Osaka Univ. of Education \\ 4-698-1 Asahigaoka, Kashiwara City, Osaka 582-8582, Japan \\ Tel. +81-729-78-3669, Fax. +81-729-78-3554 \\ e-mail:takeuti@cc.osaka-kyoiku.ac.jp
}

\begin{abstract}
We are concerned with a problem of the optimal selection of the gain matrix, over a given time interval, of a linear observation for the Kalman filter. The innovations process included in the Kalman filter has the same structure as the model of a set of parallel transmission channels with the optimal output feedback. In the linear coding problem for this set of channels, it is well-known that the optimal output feedback which minimizes the power of the encoded signal is given by the least-squares estimate of the linear term and that the channel output then becomes the innovations process. By applying a solution of the optimal transmission problem for this model, we obtain, at any time points, a set of the gains which maximize the mutual information between the observation and the signal under a constraint on the power of the innovations process. Finally, the optimal selection of the gain to minimize the estimation error is done by an optimization which is local in time.
\end{abstract}

\section{Introduction}

Starting from 1970's, the problem of optimization of observations associated with the Kalman filter has been studied in many literatures. Most of them formulated the problem as a kind of optimal control problem with a quadratic performance criterion. However, because of the existence of the nonlinearity in the Riccati equation, there are few result which is applicable in the real design of the Kalman filter.

In this paper, we apply the fact that the innovations process is described by the same equation which was used in the discussion of the optimal transmission problem of Gaussian signals through parallel channels with feedback [1]-[5]. According to the Shannon's information theory, the observation is considered to be better when the mutual information between the signal and the observation takes a larger value. On the other hand, form the view point of the performance of the Kalman filter, the observation is better when we have a smaller estimation error. Taking into account of these points, our approach in this paper is quite new and by the following two steps: (i) Information theoretic optimization to maximize the mutual information between the signal and the observation subject to a power constraint concerned with the innovations process;

(ii) Optimization of the performance of the Kalman filter, i.e., minimization of the estimation error variance.

Both (i) and (ii) are the optimization problem with respect to the observation gain matrix. Since the innovations process has the same mathematical expression as the output of parallel Gaussian channels with feedback [5], [6], we obtain a solution of (i) by applying a generalized Water Filling Theorem [6] which is the solution of the optimal transmission problem. We will show that the solution of (i) at any time point is not unique but we have a set of the gain matrices which produce the maximum value of the mutual information. In (ii), the optimal gain matrix is selected from this set of gains in such a way that the step-by-step reduction of an weighted estimation error is maximized.

A numerical example is provided to show that by a special selection of the weight matrix for a time-invariant system, the optimal solution is convergent to that of the stationary solution given by [6].

Mathematical symbols, in this paper, are used in the following way. $\boldsymbol{R}$ is the space of all real numbers, i.e., $\boldsymbol{R} \triangleq(-\infty, \infty)$. For positive integers $m$ and $n, \boldsymbol{R}^{n}$ and $\boldsymbol{R}^{m \times n}$ denote the spaces of $n$-dimensional vectors and $m \times n$-dimensional matrices whose components take values in $\boldsymbol{R}$. The prime denotes the transpose of a vector or a matrix and the Euclidean norm is $|\cdot|$. Thus, for $x \in \boldsymbol{R}^{n}, \quad|x|=\sqrt{x^{\prime} x}$. The identity matrix of any dimension is denoted by $I$ and $\operatorname{rank}[A]$ is the rank of matrix $A$ of any dimension. The components of a matrix are denoted by using subscripts. Thus, $[A]_{i j}$ is the $(i, j)$-component of $A$. In the case where no confusion may arise, we denote $[A]_{i j}$ simply by $a_{i j}$. If $A$ is a square matrix, $\operatorname{det}|A|$ and $\operatorname{tr}[A]$ respectively denote the determinant and the trace of $A$. By $\operatorname{diag}\left(a_{1}, a_{2}, \cdots, a_{n}\right)$, we denote a diagonal matrix with the diagonal components $a_{1}, a_{2}, \cdots, a_{n}$. We use $A>0$ and $A \geq 0$ to denote that $A$ is positive definite and non- 
negative definite, respectively. The triplet $(\Omega, \mathscr{\pi}, P)$ is a complete probability space where $\Omega$ is a sample space with elementary events $\omega, \mathscr{F}$ is a $\sigma$-field of subsets of $\Omega$, and $P$ is a probability measure. $E\{\cdot\}$ denotes the expectation and $E\{\cdot \mid \mathscr{E}\}, \mathscr{G} \subset \mathscr{F}$ the conditional expectation, given $\mathscr{H}$, with respect to $P . \sigma\{\cdot\}$ is the minimal sub- $\sigma$-field of $\mathscr{F}$ with respect to which the family of $\tilde{4}$-measurable sets or random variables $\{\cdot\}$ is measurable. It is assumed that all random variables and stochastic processes are $\mathscr{F}$-measurable. Unless otherwise stated, stochastic properties are that with respect to $P$.

\section{Problem Formulation and Preliminaries}

\subsection{Kalman Filter and Optimization of the Observations}

Let $x \equiv\left\{x_{t}(\omega) ; t=0,1, \cdots\right\}$ denote an $n$-dimensional Gaussian stochastic process described by

$$
x_{t+1}(\omega)=A(t) x_{t}(\omega)+G(t) w_{t}(\omega), x_{0}(\omega)=x^{0}(\omega),(1)
$$

where $A(t) \in \boldsymbol{R}^{n \times n}, \quad G(t) \in \boldsymbol{R}^{n \times d_{1}}, x^{0}(\omega)$ is a Gaussian random vector with mean $\hat{x}^{0}$ and covariance $Q^{0}$, and $w \equiv\left\{w_{t}(\omega) ; t=0,1, \cdots\right\}$ is a $d_{1}$-dimensional standard white Gaussian noise sequence. Assume that we have $\mathrm{m}$ dimensional observations described by

$$
y_{t}(\omega)=H(t) x_{t}(\omega)+R(t) v_{t}(\omega)
$$

where $\quad \boldsymbol{y} \equiv\left\{y_{t}(\omega) ; t=1,2, \cdots\right\}$ is an $m$-dimensional observation process, $H(t) \in \boldsymbol{R}^{m \times n}, \quad R(t) \in \boldsymbol{R}^{m \times d_{2}}$, and $v \equiv\left\{v_{t}(\omega) ; t=1,2, \cdots\right\}$ is a $d_{2}$-dimensional standard white Gaussian noise sequence. We will assume that

$$
\text { (C-1) } R_{0}(t) \triangleq R(t) R^{\prime}(t)>0, \quad t=1,2,3, \cdots
$$

and that $x^{0}(\omega), w$ and $v$ are mutually independent.

It is well-known that the least-squares estimate $\hat{x}_{t \mid t}(\omega) \triangleq$ $E\left\{x_{t}(\omega) \mid \mathscr{Y}_{t}\right\}$ of $x_{t}(\omega)$ based on $\mathscr{Y}_{t} \triangleq \sigma\left\{y_{s}(\omega) ; s=1,2\right.$, $\cdots, t\}$ is given by the Kalman filter:

$$
\left\{\begin{array}{l}
\hat{x}_{t \mid t-1}(\omega)=A(t) \hat{x}_{t-1 \mid t-1}(\omega) \\
\hat{x}_{t \mid t}(\omega)=\hat{x}_{t \mid t-1}(\omega)+Q_{|t|} H^{\prime}(t) R_{0}^{-1}(t) \tilde{y}_{t}(\omega),
\end{array}\right.
$$

and

$$
\left\{\begin{aligned}
Q_{t \mid t-1} & =A(t) Q_{t-1 \mid t-1} A^{\prime}(t)+G(t) G^{\prime}(t) \\
Q_{t \mid t}= & Q_{t \mid t-1}-Q_{t \mid t-1} H^{\prime}(t) \\
& \times\left\{H(t) Q_{t \mid t-1} H^{\prime}(t)+R_{0}(t)\right\}^{-1} H(t) Q_{t \mid t-1},
\end{aligned}\right.
$$

where

$$
\begin{gathered}
\hat{x}_{t \mid t-1} \triangleq E\left\{x_{t}(\omega) \mid \mathscr{Y}_{t-1}\right\}, \\
Q_{t \mid t-1} \triangleq E\left\{\left[\hat{x}_{t \mid t-1}(\omega)-x_{t}(\omega)\right]\left[\hat{x}_{t \mid t-1}(\omega)-x_{t}(\omega)\right]^{\prime}\right\}, \\
Q_{t \mid t} \triangleq E\left\{\left[\hat{x}_{|| t}(\omega)-x_{t}(\omega)\right]\left[\hat{x}_{t \mid t}(\omega)-x_{t}(\omega)\right]^{\prime}\right\} .
\end{gathered}
$$

Also, $\tilde{y} \equiv\left\{\tilde{y}_{t}(\omega) ; t=1,2, \cdots\right\}$ in (3) is the innovations process:

$$
\begin{aligned}
\tilde{y}_{t}(\omega) & \triangleq y_{t}(\omega)-H(t) \hat{x}_{t \mid t-1}(\omega) \\
& =H(t)\left\{x_{t}(\omega)-\hat{x}_{t \mid t-1}(\omega)\right\}+R(t) v_{t}(\omega) .
\end{aligned}
$$

Let $T \in\{1,2,3, \cdots\}$ be any finite time point. In this paper, the optimization of $\boldsymbol{H}_{0}^{T} \equiv\{H(t) ; t=1,2, \cdots, T\}$ is done by the following two steps.

(i) Optimization by an information theoretic criterion: Find a set, say $\mathscr{H}_{T}$, of the values of $\boldsymbol{H}_{0}^{T}$ which maximize the mutual information between $x$ and $y$ subject to a power condition concerned with the innovations process.

(ii) Find $\boldsymbol{H}_{0}^{T} \in \mathscr{H}_{T}$ which minimizes a scalar-valued function of $Q_{T \mid T}$ or $Q_{t \mid}, 1 \leq t \leq T$.

As for (i), the problem in the optimal transmission framework is described as follows [5], [6].

[Problem 1 (Optimal Selection (i) of $\boldsymbol{H}_{0}^{T}$ )] Find $\boldsymbol{H}_{0}^{T}$ $\equiv\{H(t) ; t=1,2, \cdots, T\}$ such that

$$
\begin{gathered}
I_{T}(x, y)=\sum_{t=1}^{T} \Delta I_{t}(x, y) \rightarrow \max . \\
\Delta I_{t}(x, y) \triangleq \frac{1}{2} \log \left\{\frac{\operatorname{det}\left|H(t) Q_{t \mid t-1} H^{\prime}(t)+R_{0}(t)\right|}{\operatorname{det}\left|R_{0}(t)\right|}\right\},
\end{gathered}
$$

subject to the power condition:

$\Delta P_{t}^{*} \triangleq \operatorname{tr}\left[H(t) Q_{t \mid t-1} H^{\prime}(t)\right] \leq p(t), \quad t=1,2, \cdots, T$

where $p(t)$ is a maximum admissible value.

In the next subsection, we will give some explanations about the optimal transmission problem and how we get the innovations process and Problem 1 in it.

\subsection{Optimization of $\boldsymbol{H}_{0}^{T}$ from an Information Theoretic Point of View}

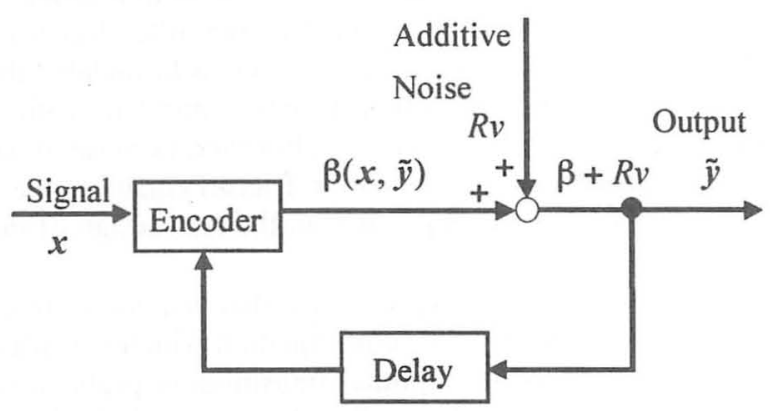

Fig. 1 Transmission channels with feedback.

From (8), we notice that the innovations process $\tilde{y}$ is described by the same equation which was used in the discussion of the optimal transmission problem [1]. The optimal transmission problem for a set of parallel transmission channels with the construction shown in Fig. 1 is 
described as follows. Here, we will also assume that $x \equiv\left\{x_{t}(\omega) ; t=1,2, \cdots\right\}$ which denotes the signal is an $n-$ dimensional Gaussian stochastic process given by (1).

In what follows, on the other hand, we temporary reset the definition of $\tilde{y}$ to denote the output of the channels. When we send $x$ through the set of $m$-parallel channels, we employ an encoding scheme. Namely, we send an encoded signal $\beta \equiv\{\beta,(x, \tilde{y}) ; t=1,2, \cdots\}$ which is a function of both signal $x$ and output $\tilde{y} \equiv\left\{\tilde{y}_{t}(\omega)\right.$; $t=1,2, \cdots\}$. Thus, the output of the transmission channels in Fig. 1 is described by

$$
\tilde{y}_{t}(\omega)=\beta_{t}(x, \tilde{y})+R(t) v_{t}(\omega) .
$$

An optimal transmission problem, or an optimal coding problem, is the one to determine $\beta$ optimally. Let us consider a linear coding problem where $\beta$ is given by

$$
\beta_{t}(x, \tilde{y})=H(t) x_{t}(\omega)-\phi(t, \tilde{y}) .
$$

Since $\phi(t, \tilde{y})$ must be a non-anticipative function of $\tilde{y}$, we have

$$
\phi(t, \tilde{y})=\phi\left(t, \tilde{y}_{1}, \cdots, \tilde{y}_{t-1}\right),
$$

namely, the feedback component $\phi(t, \tilde{y})$ must be a $\tilde{\mathscr{Y}}_{t-1}$ measurable random vector, where

$$
\tilde{y}_{t} \triangleq \sigma\left\{\tilde{y}_{s}(\omega) ; s=1,2, \cdots, t\right\} .
$$

Note that if $\phi(t, \tilde{y}) \equiv 0$, we have $y$ given by (2) as the output of the channels. As it is well-known [3], $\phi(t, \tilde{y})$ does not affect the information given by $\tilde{y}$.

«Theorem 1» Assume (C-1). Then, we have the informational equivalence between $\tilde{y}$ and $y$, i.e., we have

$$
\tilde{\mathscr{Y}}_{t}=\mathscr{Y}_{t}, \quad t=1,2, \cdots .
$$

Let $I_{t}(x, \tilde{y})$ denote the mutual information between $x^{\prime} \equiv\left\{x_{s}(\omega) ; s=0,1, \cdots, t\right\} \quad$ and $\quad \tilde{y}^{\prime} \equiv\left\{\tilde{y}_{s}(\omega) ; s=1,2\right.$, $\cdots, t\}$. Then, we have the following property on $I_{1}(\boldsymbol{x}, \tilde{\boldsymbol{y}})$ and $\phi(s, \tilde{\boldsymbol{y}}), s \leq t$.

«Theorem 2» $I_{t}(\boldsymbol{x}, \tilde{\boldsymbol{y}})$ does not depend on $\phi(s, \tilde{\boldsymbol{y}})$, $s=1,2, \cdots, t$, i.e., $I_{t}(\boldsymbol{x}, \tilde{\boldsymbol{y}}) \equiv I_{t}(\boldsymbol{x}, \boldsymbol{y})$ and $\Delta I_{t}(\boldsymbol{x}, \tilde{\boldsymbol{y}}) \equiv$ $\Delta I_{t}(x, y)$.

Let $P_{t}$ denote the transmission power up to $t$, i.e.,

$$
P_{t} \triangleq \sum_{s=1}^{t} E\left\{\left|\beta_{s}(x, y)\right|^{2}\right\}
$$

Also, for the accumulated values given by (17), let $\Delta P_{t}$ denote the value at one time point $t$, i.e.,

$$
\Delta P_{t} \triangleq E\left\{|\beta,(x, y)|^{2}\right\}
$$

From the information theoretic point of view, it is desirable to select $\beta$ in such a way that we have output $\tilde{y}$ for which $I_{T}(x, \tilde{y})$ takes a value as large as possible. On the other hand, it is usually the case that $\Delta P_{t}$ for each $t \in\{1,2, \cdots, T\}$ cannot exceed a value, say $p(t)$, which is determined by the restriction on the transmission channels. Then, the optimal transmission problem is described by

[Optimal Transmission Problem] Find $\beta_{t}(x, \tilde{y}), t=$ $1,2, \cdots, T$ such that

$$
I_{T}(\boldsymbol{x}, \tilde{y})=\sum_{t=1}^{T} \Delta I_{t}(\boldsymbol{x}, \tilde{y}) \rightarrow \max .,
$$

subject to the power condition:

$$
\Delta P_{t} \leq p(t), \quad t=1,2, \cdots, T,
$$

where $p(t)$ is the maximum admissible transmission power at time $t$.

Now, by (13), the optimal transmission problem is that of finding $\boldsymbol{\varphi}_{0}^{T} \equiv\{\phi(t, \tilde{\boldsymbol{y}}) ; t=1,2, \cdots, T\} \quad$ and $\quad \boldsymbol{H}_{0}^{T} \equiv$ $\{H(t) ; t=1,2, \cdots, T\}$ for which we have (19) and (20). By Theorem 2, and by the duality of (19) and (20), the problem is divided into the following two sub-problems:

[Problem S1] For a fixed $\boldsymbol{H}_{0}^{T}$, find $\boldsymbol{\varphi}_{0}^{T}$ such that

$$
P_{T}=\sum_{t=1}^{T} \Delta P_{t} \rightarrow \min .
$$

[Problem S2] For the solution $\varphi_{0}^{T}=\varphi_{0}^{* T}$ of Problem S1, find $\boldsymbol{H}_{0}^{T}$ such that

$$
I_{T}(x, \tilde{y})=\sum_{i=1}^{T} \Delta I_{t}(x, \tilde{y}) \rightarrow \max .,
$$

subject to the power condition:

$$
\Delta P_{t} \leq p(t), \quad t=1,2, \cdots, T .
$$

As for Problem S1, it can be seen from (17) and (13) that $\varphi_{0}^{T} \equiv\{\phi(t, \tilde{y}) ; t=1,2, \cdots, T\}$ is optimal when

$$
P_{T}=\sum_{t=1}^{T} \Delta P_{t}=\sum_{t=1}^{T} E\left\{\left|H(t) x_{t}(\omega)-\phi(t, \tilde{y})\right|^{2}\right\},
$$

is minimized. According to the least-squares estimation theory, the optimal selection of $\varphi_{0}^{T}$ is given by the following theorem.

«Theorem 3» The solution of Problem S1 is given by

$$
\phi(t, \tilde{\boldsymbol{y}})=H(t) \hat{x}_{t \mid t-1}(\omega), \quad t=1,2, \cdots, T,
$$

where $\hat{x}_{t \mid t-1}(\omega)$ is the prediction of $x_{t}(\omega)$ defined by (5). When (23) is used, we have

$$
\Delta P_{t}=\operatorname{tr}\left[H(t) Q_{\mid t-1} H^{\prime}(t)\right], \quad t=1,2, \cdots, T .
$$

If we take $\phi(t, \tilde{y})$ by (23), we see from (12) and (13) that the output of the transmission channels is described by (8), i.e., the channel output is now the innovations process. Also, since $I_{t}(x, \tilde{y}) \equiv I_{t}(x, y)$ by Theorem 2 , it is seen 
that Problem S2 is reduced to Problem 1 in the previous subsection.

\section{Optimal Selection of $H(t)$ by way of a \\ Solution of Optimal Transmission Problem}

As we explained in the first part of the previous section, the optimization of $\boldsymbol{H}_{0}^{T}$ has two steps. For the first step, i.e., for Problem 1, the condition of the optimality is given by the following two theorems [6].

\section{«Theorem 4 (A Generalized Water Filling Theorem)»} Assume (C-1) and

(C-2) $G(t) G^{\prime}(t)>0$ for all $t \in\{1,2, \cdots, T\}$.

Let $\quad\left(\psi_{1}(t), \psi_{2}(t), \cdots, \psi_{m}(t)\right) \quad$ and $\quad\left(\gamma_{1}(t), \gamma_{2}(t), \cdots\right.$, $\left.\gamma_{m}(t)\right)$ denote the set of eigen-values of $R_{0}(t)$ and the corresponding set of eigen-vectors, respectively. Also, let $\Psi(t) \triangleq \operatorname{diag}\left(\psi_{1}(t), \psi_{2}(t), \cdots, \psi_{m}(t)\right)$ and $\Gamma(t) \triangleq\left[\gamma_{1}(t)\right.$ $\left.\gamma_{2}(t) \cdots \gamma_{m}(t)\right]$, i.e., $R_{0}(t)=\Gamma(t) \Psi(t) \Gamma^{\prime}(t)$. Without loss of generality, we can assume that

$$
0<\psi_{1}(t) \leq \psi_{2}(t) \leq \cdots \leq \psi_{m}(t) .
$$

Then, for Problem 1, the condition of optimality of $H(t)$ $\in \boldsymbol{R}^{m \times n}, t=1,2, \cdots, T$ is given by

$$
H(t) Q_{t \mid t-1} H^{\prime}(t)=\Gamma(t) \Xi(t) \Gamma^{\prime}(t),
$$

where

$$
\begin{gathered}
\Xi(t) \triangleq \operatorname{diag}\left(\xi_{1}(t), \xi_{2}(t), \cdots, \xi_{m}(t)\right), \\
\xi_{i}(t) \triangleq \max \left\{0, \alpha(t)-\psi_{i}(t)\right\}, \quad i \in\{1,2, \cdots, m\} \\
\alpha(t) \triangleq \frac{p(t)+\sum_{i=1}^{\tilde{m}(t)} \psi_{i}(t)}{\tilde{m}(t)},
\end{gathered}
$$

and

$$
\begin{aligned}
\tilde{m}(t) & \triangleq \operatorname{rank}[\Xi(t)] \\
& =\left\{\text { Number of positive } \xi_{i}(t), 1 \leq i \leq m\right\} .
\end{aligned}
$$

Furthermore, for any $H(t)$ given by (26), we have

$$
H(t) Q_{t \mid t} H^{\prime}(t)=\Gamma(t) \Xi(t)\{\Xi(t)+\Psi(t)\}^{-1} \Psi(t) \Gamma^{\prime}(t) .
$$

and

$$
I_{T}(\boldsymbol{x}, \boldsymbol{y})=\frac{1}{2} \sum_{t=1}^{T} \log \left\{\operatorname{det}\left|\Xi(t) \Psi^{-1}(t)+I\right|\right\} .
$$

[Remark 1] It should be noted that $H(t) Q_{t \mid t-1} H^{\prime}(t)$ and $R_{0}(t)$ have the same set of eigen-vectors and the sum of the pair of eigen-values $\xi_{i}(t)$ and $\psi_{i}(t)$ corresponding to each eigen-vector $\gamma_{i}(t)$ is equal to $\alpha(t)$ if $\psi_{i}(t)<\alpha(t)$. Otherwise, i.e., if $\psi_{i}(t) \geq \alpha(t)$, we have $\xi_{i}(t)=0$. This is the same result as the well-known Water Filling Theorem ${ }^{[6]}$ except for the point that our result is concerned with the eigen-values of the signal and the noise covariance matrices whereas the usual case is with the signal and the noise powers for channels.

[Remark 2] Due to (25), we have

$$
\begin{aligned}
\xi_{1}(t) & \geq \xi_{2}(t) \geq \cdots \geq \xi_{\tilde{m}(t)}(t) \\
& >\xi_{\tilde{m}(t)+1}(t)=\cdots=\xi_{m}(t)=0 .
\end{aligned}
$$

[Remark 3] The maximum value of the mutual information given by (32) only depends on $\Psi(t)$ and $p(t)$ because $\alpha(t)$ and $\Xi(t)$ are determined by them. Thus, the mutual information is independent of the stochastic property of the signal except for the condition that the mean squares power of $H(t) x_{t}(\omega)$ is $p(t)$.

[Remark 4] In order to determine the values of $\alpha(t)$ and $\tilde{m}(t)$, we can use a recursive algorithm [6].

[Remark 5], It should be noted that $\Xi(t), \Psi(t)$ and $\{\Psi(t)+\Xi(t)\}^{-1}$ in (31) are all diagonal matrices, and that they are commute in matrix multiplication.

«Theorem 5 (Reduction of Riccati Equation)» Assume (C-1) and (C-2). For simplicity, let us denote

$$
\begin{gathered}
\Xi(t)=\left[\begin{array}{cc}
\tilde{\Xi}(t) & 0 \\
0 & 0
\end{array}\right], \\
\tilde{\Xi}(t)=\operatorname{diag}\left(\xi_{1}(t), \xi_{2}(t), \cdots, \xi_{\tilde{m}(t)}(t)\right), \\
\xi_{i}(t)>0, \quad i \in\{1,2, \cdots, \tilde{m}(t)\}
\end{gathered}
$$

and

$$
\begin{aligned}
& \Gamma(t)=\left[\begin{array}{ll}
\tilde{\Gamma}(t) & \bar{\Gamma}(t)
\end{array}\right], \\
& \tilde{\Gamma}(t) \in \boldsymbol{R}^{m \times \tilde{m}(t)}, \quad \bar{\Gamma}(t) \in \mathbb{R}^{m \times(m-\tilde{m}(t))} .
\end{aligned}
$$

Then, the solution of Problem 1 is given by the following set of equations.

$$
\begin{aligned}
Q_{t+|| t} & =A(t) Q_{t \mid t} A^{\prime}(t)+G(t) G^{\prime}(t) . \\
Q_{t \mid t} & =\left(Q_{t \mid t-1}\right)^{1 / 2} K(t)\left(Q_{t \mid t-1}\right)^{1 / 2}, \\
K(t) & \triangleq I-\frac{1}{\alpha(t)} \tilde{U}(t) \tilde{\Xi}(t) \tilde{U}^{\prime}(t), \\
H(t) & =\tilde{\Gamma}(t) \tilde{\Xi}^{1 / 2}(t) \tilde{U}^{\prime}(t)\left(Q_{t \mid t-1}\right)^{-1 / 2},
\end{aligned}
$$

where $\tilde{U}(t) \in \boldsymbol{R}^{n \times \tilde{m}(t)}$ is any matrix with the property

$$
\tilde{U}^{\prime}(t) \tilde{U}(t)=I \text {. }
$$

[Remark 6] From (36)-(40), it is clear that any observation with $\boldsymbol{H}_{0}^{T}$ given by (39) and the same value of $\tilde{U}(t)$ produce the same filtering performance. Thus, the performance of the Kalman filter under (39) depends on $\Psi(t), p(t)$ and $\tilde{U}(t)$ other than $A(t)$ and $G(t)$, and is independent of $\Gamma(t)$.

\section{Local Optimization of $\tilde{U}(t), 1 \leq t \leq T$.}

By (39), it is seen that $\boldsymbol{H}_{0}^{T}$ is not unique but we can take 
any $\tilde{U}(t) \in \boldsymbol{R}^{n \times \tilde{m}(t)}$ with property: $\tilde{U}^{\prime}(t) \tilde{U}(t)=I$. Namely, $\mathscr{H}_{T} \triangleq\left\{\boldsymbol{H}_{0}^{T} ; H=\tilde{\Gamma} \tilde{\Xi}^{1 / 2} \tilde{U}^{\prime} Q_{(\cdot)(\cdot)-1}^{-1 / 2}, \tilde{U}^{\prime} \tilde{U}=I, 0 \leq t \leq T\right\}$,

is the set of solutions of Problem 1. From the view point of the performance of the Kalman filter, it is desirable to select $\tilde{U}(t), 0 \leq t \leq T$ such a way as to minimize a scalar-valued function of $Q_{T \mid T}$ or $Q_{t \mid t}, 1 \leq t \leq T$. The solution of this step, (ii), of the problem is, however, very difficult because it is a multi-stage optimization problem with a nonlinear dynamics.

For this reason, we will use the following local optimization scheme to determine $\tilde{U}(t), 0 \leq t \leq T$.

[Local Optimization Problem] For each $t \in\{1,2, \cdots$, $T\}$, let $M(t) \in R^{n \times n}$ be a nonnegative definite symmetric matrix with positive eigenvalues more than or equal to $\tilde{m}(t)=\operatorname{rank}[H(t)] . \quad$ Under (36)-(38) and (40), we determine $\tilde{U}(t)$ such that

$$
\operatorname{tr}\left[M(t) Q_{t \mid r}\right] \rightarrow \min .
$$

The solution of the above local optimization (LOOP) problem is given by the following theorem.

«Theorem 6 (Local Optimization of $\tilde{U}$ )» Assume (C-1) and $(\mathrm{C}-2)$. Let us define Lagrangean by

$$
L(t, \Lambda, \tilde{U}) \triangleq \operatorname{tr}\left[M(t) Q_{t \mid r}\right]+\operatorname{tr}\left[\Lambda(t)\left(\tilde{U}^{\prime}(t) \tilde{U}(t)-I\right)\right]
$$

where $\Lambda(t)$ is a symmetric matrix with the Lagrange multipliers $\lambda_{i j}(t), 1 \leq i, j \leq \tilde{m}(t)$ as its components. Then,

(i) A condition of optimality of $\tilde{U}(t)$ is given by

$$
Q_{t \mid t-1}^{1 / 2} M(t) Q_{t \mid t-1}^{1 / 2} \tilde{U}(t)=\tilde{U}(t) \alpha(t) \Lambda(t) \tilde{\Xi}^{-1}(t) .
$$

(ii) For (42), we can take $\Lambda(t)$ as a diagonal matrix.

(iii) $\tilde{U}(t)$ is optimal when we take the columun vectors of it in such a way that the diagonal components of $\tilde{U}^{\prime}(t) Q_{t \mid t-1}^{1 / 2} M(t) Q_{t \mid t-1}^{1 / 2} \tilde{U}(t)$ is in descending order.

[Remark 7] From (42), we see that the $i$ th columun vector of $\tilde{U}(t)$ is an eigenvector of $Q_{t \mid t-1}^{1 / 2} M(t) Q_{t \mid t-1}^{1 / 2}$ and the corresponding eigenvalue is the $i$ th diagonal component of $\alpha(t) \Lambda(t) \tilde{\Xi}^{-1}(t)$.

For the performance and an asymptotic property of the LOOP algorithm, we will give a numerical example in Section 6.

\section{Proofs of Theorems}

In this section, we will give proofs of the theorems presented in the previous section.

(Proof of Theorem 4) By [5; Theorem 3.1], we have

$$
\begin{aligned}
\Delta I_{T}^{*}(x, y) & \triangleq \max _{\substack{H(T) \\
\Delta P_{T}^{*} \leq p(T)}} \Delta I_{T}(x, y) \\
& =\frac{1}{2} \log \left\{\operatorname{det}\left|\Xi(T) \Psi^{-1}(T)+I\right|\right\},
\end{aligned}
$$

and (26)-(31) for $t=T$. Then, for

$$
I_{T}^{*}(x, y) \triangleq \max _{\substack{H_{0}^{T} \\ \Delta P_{1}^{*}>p(t) \\ 1 \leq t \leq T}} I_{T}(x, y),
$$

we have the relation

$$
I_{T}^{*}(x, y)=I_{T-1}^{*}(x, y)+\frac{1}{2} \log \left\{\operatorname{det}\left|\Xi(T) \Psi^{-1}(T)+I\right|\right\} .
$$

Thus, $\Delta I_{T}^{*}(\boldsymbol{x}, \boldsymbol{y})$ is independent of $\boldsymbol{H}_{0}^{T-1}$. Hence, by repeating the sigle-stage optimization of $\Delta I_{t}(x, y), t=$ $T-1, T-2, \cdots, 2,1$, we have (26)-(31) and (32).

( Proof of Theorem 5 ) The proof of this theorem is simply the combination of those of [5; Theorem 3.2] and [6; Theorem 3.2].

( Proof of Theorem 6 ) Substituting (37) and (38) into $L(t, \Lambda, \tilde{U})$ and differentiating it with respect to $\tilde{U}(t)$, we have

$$
\frac{\partial L}{\partial \tilde{U}}=-\frac{2}{\alpha(t)} Q_{t \mid t-1}^{1 / 2} M(t) Q_{t \mid t-1}^{1 / 2} \tilde{U}(t) \tilde{\Xi}(t)+2 \tilde{U}(t) \Lambda(t)=0,
$$

from which we have (42). The proof of (ii) and (iii) are the same as [6; Theorem 3.3].

\section{A Numerical Example}

In this section, we will give an illustrative example for a 2-dimensional system for signal $\boldsymbol{x}(n=2)$ and 2dimensional observations $(m=2)$.

Example 1. Let us consider a 2-dimensional system with

$$
A(t) \equiv A=\left[\begin{array}{ll}
0.5 & 0.3 \\
0.2 & 0.4
\end{array}\right], \quad G(t) \equiv G=\left[\begin{array}{cc}
0.5 & 0 \\
0 & 0.5
\end{array}\right] .
$$

We assume

$$
Q^{0}=Q_{0 \mid 0}=\left[\begin{array}{cc}
1.5 & 0 \\
0 & 1.5
\end{array}\right] .
$$

Consider 2-dimensional observations with the following parameters:

$$
\begin{aligned}
R_{0}(t) & \equiv R_{0}=\left[\begin{array}{cc}
\frac{\sqrt{3}}{2} & \frac{1}{2} \\
-\frac{1}{2} & \frac{\sqrt{3}}{2}
\end{array}\right]\left[\begin{array}{cc}
0.49 & 0 \\
0 & 1.0
\end{array}\right]\left[\begin{array}{cc}
\frac{\sqrt{3}}{2} & -\frac{1}{2} \\
\frac{1}{2} & \frac{\sqrt{3}}{2}
\end{array}\right] \\
& =\left[\begin{array}{ll}
0.6175 & 0.2208 \\
0.2208 & 0.8725
\end{array}\right],
\end{aligned}
$$

i.e.,

$$
\Psi(t) \equiv \operatorname{diag}(0.49,1.0), \quad \Gamma(t)=\left[\begin{array}{cc}
\frac{\sqrt{3}}{2} & \frac{1}{2} \\
-\frac{1}{2} & \frac{\sqrt{3}}{2}
\end{array}\right] .
$$


For the above system and observations, we made numerical computations for two cases of the value of $p(t) \equiv p$, i.e., $p=1.2$ and $p=0.4$. For these cases, the values of $\tilde{m}(t), \alpha(t), \tilde{\Psi}(t), \tilde{\Xi}(t)$ and $\Delta I_{t}(x, y)$ are all constant in $t$ and are summarized in Table 1.

Table 1. The values of $\tilde{m}(t), \alpha(t), \quad \tilde{\Psi}(t), \quad \tilde{\Xi}(t)$ and $\Delta I_{t}(\boldsymbol{x}, \boldsymbol{y})$ for $p(t) \equiv 1.2$ and $p(t) \equiv 0.4$.

\begin{tabular}{|c|c|c|c|c|c|}
\hline$p$ & $\tilde{m}$ & $\alpha$ & $\tilde{\Psi}$ & $\tilde{\Xi}$ & $\Delta I_{t}(x, y)$ \\
\hline 1.2 & 2 & 1.345 & $\operatorname{diag}(0.49,1.0)$ & $\operatorname{diag}(0.855,0.345)$ & 0.6531 \\
\hline 0.4 & 1 & 0.890 & 0.49 & 0.40 & 0.2984 \\
\hline
\end{tabular}

Here we should note that for $p(t)=0.4$, we have $\tilde{m}(t)=1$ and $\xi_{2}(t)=0$ since $\psi_{2}(t)=1.0>0.89=\alpha(t)$ This implies that we cannot assign, due to the constraint $p(t)=0.4$, any energy for the second eigen-vector component in $\Gamma(t)$ of $H(t) Q_{t \mid t-1} H^{\prime}(t)$ because this component is very much corrupted by the observation noise $\left(\psi_{2}(t)=1.0\right)$. Thus, for $p(t)=0.4, \tilde{U}(t) \in$ $\boldsymbol{R}^{2 \times 1}$, namely, $\tilde{U}(t)$ is a two-dimensional vector, and the degree of freedom of $H(t) \in \boldsymbol{R}^{2 \times 2}$ is restricted to that of a two-dimensional vector, i.e., $\operatorname{rank}[H(t)]=1$.

The above parameters are the same as that of the numerical example given in [6] for the stationary case. Based on (42) and the corresponding condition of optimality in a similar form as in [6], we take

$$
M(t) \equiv D-A^{\prime} \Theta A=\left[\begin{array}{cc}
1.136861746 & 0.0961965831 \\
0.0961965831 & 1.116491803
\end{array}\right],
$$

where $D$ and $\Theta$ are the values in the solution of the stationary problem (See [6] for detail). It is seen that for the above value of $M(t)$, the solution of the stationary problem is an equilibrium point of $\left(\tilde{U}(t), H(t), Q_{t \mid t-1}, Q_{t \mid t}\right)$ in the LOOP algorithm. The results of the LOOP algorithm

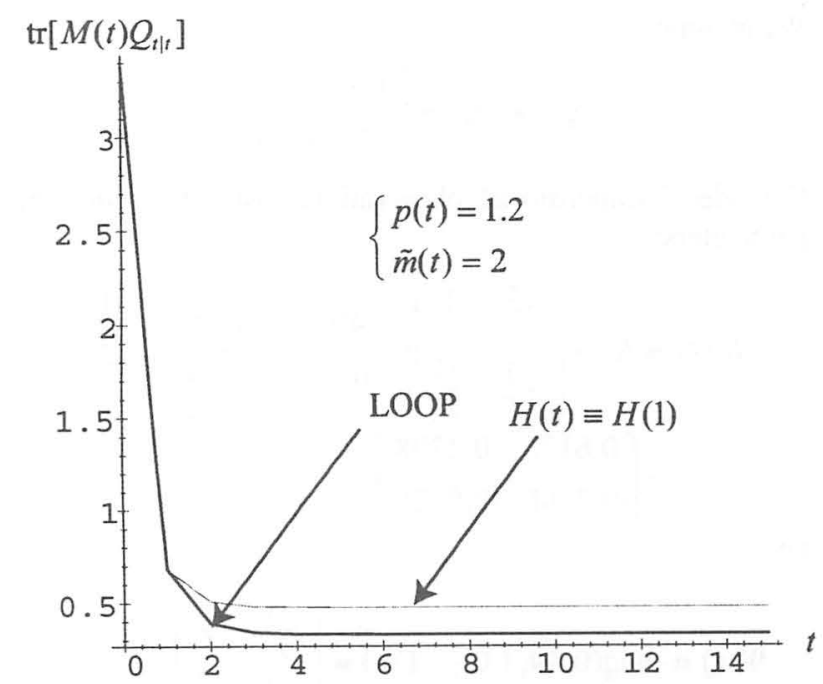

Fig. 2 Time-evolution of $\operatorname{tr}\left[M(t) Q_{t \mid t}\right]$ for $p(t) \equiv 1.2$.

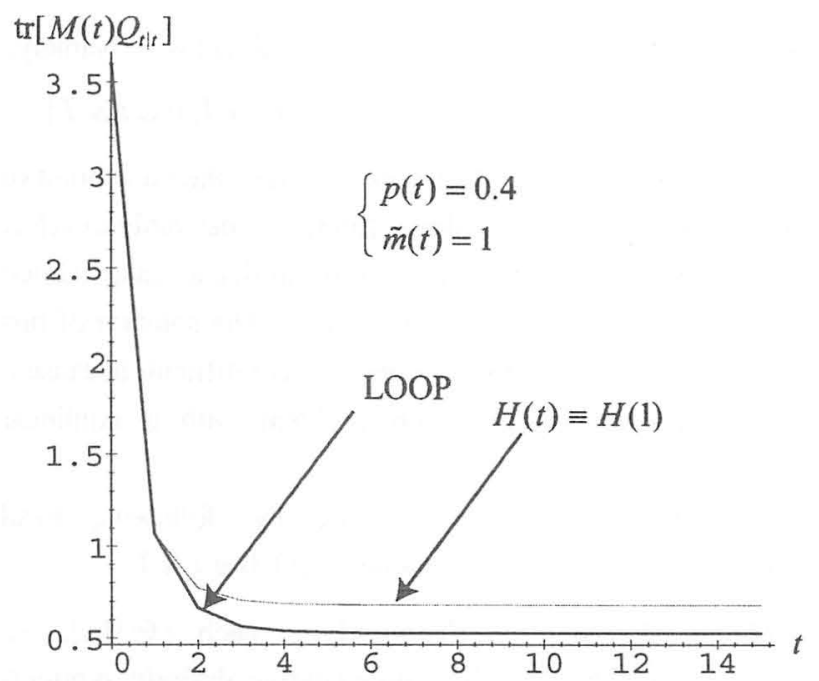

Fig. 3 Time-evolution of $\operatorname{tr}\left[M(t) Q_{t \mid t}\right]$ for $p(t) \equiv 0.4$.

for $p(t) \equiv 1.2$ and $p(t) \equiv 0.4$ are given in Fig. 2 and Fig. 3. From them, we notice that if we take $M(t)$ by (46), then the solution tends to an equilibrium point which is a solution of the sationary case of the problem.

\section{Concluding Remarks}

In this paper, we have presented an information theoretic approach to the optimization of observations for Kalman filter. By the LOOP algorithm to determin $\tilde{U}(t)$ in Section 4 and the method of determining $M(t)$ in Section 6 , the convergence to the stationary solution is also seen in the numerical study.

\section{References}

[1] S. Ihara, "Coding theory in white Gaussian channel with feedback," J. Multi-variate Analysis, 4, 74/87, 1974.

[2] Y. Takeuchi, "The optimal transmission of non-Gaussian signals through a noisy channel with feedback," IEEE Trans. Inform. Theory, IT-40, 5, 1624/1629, 1994.

[3] Y. Takeuchi and A. Ida, "Optimal Transmission of a set of Gaussian Signals through Discrete-Time Channels with Feedback," Proc. of 35th ISCIE SSS, Ube, Oct. 30-31, 2003, 118/123, 2004.

[4] Y. Takeuchi, "An information theoretic approach to optimization of linear observations for the Kalman-Bucy filter," International Journal of Innovative Computing, Information \& Control, 1-3, 401/416, 2005.

[5] Y. Takeuchi, "Optimal transmission of a set of discretetime Gaussian signals through channels with feedback," International Journal of Innovative Computing, Information \& Control, 2-5, 927/942, 2006.

[6] Y. Takeuchi, " Optimization of linear observations for the stationary Kalman filter based on a generalized Water Filling Theorem," International Journal of Innovative Computing, Information \& Control, 4-1, 211/230, 2008. 